\title{
A study on incidence of gastrointestinal parasites in buffaloes from different slaughter houses of Chittagong Metropolitan Area
}

\author{
Mohammad Belayet Hossain ${ }^{1}$, Md. Selim Mahmud ${ }^{1}$, Shubaghata Das ${ }^{2}$, Md. Abdul Alim², Amir Hossan \\ Shaikat $^{1}$ and Md. Abul Hashem ${ }^{3}$ \\ ${ }^{1}$ Department of Physiology, Biochemistry and Pharmacology, ${ }^{2}$ Department of Pathology and Parasitology, \\ Chittagong Veterinary and Animal Sciences University, Khulshi, Chittagong-4202. ${ }^{3}$ Department of Health, \\ Chittagong City Corporation, Chittagong.
}

\begin{abstract}
The study was conducted to investigate the incidence of gastrointestinal parasites of buffalo in different slaughter houses of Chittagong Metropolitan Area, Bangladesh. A total of 100 faecal samples were collected from three slaughter houses and studied by coproscopic examinations. Direct smear, sedimentation and floatation techniques along with egg micrometry were conducted to identify various parasitic eggs. Among 100 faecal samples 69\% were positive for different individual parasitic infestation. Trematodes, nematodes, cestodes and protozoan infestations were 48\%, 16\%, 2\% and 3\%, respectively. Among the trematodes Schistosoma sp. (23\%), Paramphistomum sp. (15\%) and Fasciola sp. (10\%) were found. Incidence of nematode infestations were Toxocara sp (4\%), Oesophagostomum sp. (5\%), Cooperia sp. (2\%), Bunostomum sp. (3\%) and $1 \%$ for both Trihostrongylus sp. and Strongyloides sp. Mixed infection were observed $7 \%$ of positive samples. Results indicate that the heavy worm burden in buffaloes causes severe economic loss to the farmers by reducing the overall productivity.
\end{abstract}

Key Words: Slaughter house, buffalo, coproscopy, parasites.

\section{Introduction}

Livestock has been an essential component of the farming systems of a country. Buffaloes are one of the most important domestic animals which produce meat, higher percentages fat containing milk, manure and draught power in Bangladesh. There are some factors which affect the production performance of buffaloes. Among them parasites are most important factors causing great economic losses in terms of mortality and decreased milk and meat production (Raza, 2007). The helminthes parasitism, especially gastrointestinal parasitism, is one of the major health problems for draught and dairy buffaloes (Shan and Chaudhry, 1995). Buffaloes exposed to a higher risk of infection with snail borne helminthes due to the animals' propensity to seek rivers, pools or swamps for wallowing. Some helminthes of buffaloes are also transmissible directly or indirectly to humans where they can cause significant clinical diseases such as schistosomiasis, hydatidosis, echinococcosis and fascioliasis in a number of countries including Bangladesh (Tum et al., 2007). Keeping these facts in mind, the present study was undertaken to investigate the incidence of gastrointestinal parasites in buffalo samples collected from different slaughter houses of Chittagong Metropolitan Area.

\section{Materials and Methods}

Study area: Buffalo faeces sample were collected from different slaughter houses in Chittagong Metropolitan Area and brought to the Parasitology
Laboratory of Chittagong Veterinary and Animal Sciences University for further analyses.

Sampling technique and sample size: A total of 100 faecal samples of buffaloes were collected from the slaughter houses of Firingi bazar, Pahartali and Oxygen of Chittagong Metropolitan Area. The buffaloes were gathered from the border areas of India and costal areas and kept for sale or slaughter purpose. With the help of flotation and sedimentation technique, these samples were examined. These samples were collected from pre-slaughtered animals. To ensure better condition during sample collection the following precautions were taken. The fresh faecal samples were collected in air-tight containers to prevent desiccation, $4-5$ drops of $10 \%$ formalin were used to fix the samples and refrigerated until used.

Examination of samples: The faecal samples were examined by direct smear, flotation and sedimentation techniques and faeces counting method. The species of the various eggs of the parasites were identified according to their characters and morphology.

Direct smear method: A drop of water was taken on a glass slide. A small amount of faeces was spread out and a thin smear was made. The coarse particles were discarded gently. Then a cover slip was placed over the smear.

Differential flotation technique: 2-3gm of faecal sample was taken in a beaker to which $40 \mathrm{ml}$ water 
was added. With the help of a spatula, the sample was stirred and filtered with a tea strainer. The filtered sample $(15 \mathrm{ml})$ was then poured into plastic test tube and centrifuged at $1500 \mathrm{rpm}$ for $5 \mathrm{~min}$. The tube was taken out and the upper part of the water was removed with the help of a dropper. The tube was filled with sodium chloride solution and centrifuged at $1500 \mathrm{rpm}$ for $5 \mathrm{~min}$. More sodium chloride was added up to the tip of the tube. A cover slip was placed over the top of the tube so that the sodium chloride touches the cover slip for a few minutes and then the cover slip was placed on a slide and examined at $10 x$ to $40 x$ under microscope.

Sedimentation technique: 2-3gm of faecal sample was taken in a beaker to which $40 \mathrm{ml}$ water was added. The sample was mixed properly and then the sample was filtered using a tea strainer and the filtered sample was poured in a plastic test tube and centrifuged at $1500 \mathrm{rpm}$ for $5 \mathrm{~min}$. The tube was taken out and the upper part of water was removed with the help of a dropper. Sodium chloride solution was taken in the test tube and again centrifuged at $1500 \mathrm{rpm}$ for $5 \mathrm{~min}$. A drop of the deposited materials was taken out from the test tube with the dropper and placed on the slide, and finally examined the slide under microscope at $10 x$ or $40 x$.

Faeces counting method: It is the easiest quantitative method to count the number of eggs present in the faeces without the help of McMaster. Species-wise eggs of the helminthes parasites viz. trematodes, nematodes and cestodes, were observed and counted. The number of eggs determined the number of eggs present per gm of the faecal matter.

Statistical analysis: Data were entered into a spreadsheet of Microsoft Excel and were analyzed using Graph pad software.

\section{Results and Discussion}

Incidence of parasites in buffaloes: Sixty nine percent of all buffaloes were infested with different gastrointestinal parasites (Fig. 1).

Incidence of trematodes: The incidence of trematode infestation of buffaloed (Table 1) was statistically very significant $(P<0.01)$. The highest incidence was of Schistosoma sp. (23\%) followed by Paramphistomum sp. (15\%) and Fasciola sp.. $(10 \%)$. This result supports the earlier reports by Kabir et al. (2010) and Shaikh et al. (1983) who observed $10 \%$ incidences each of Paramphistomum sp. and Fasciola $\mathrm{sp}$. in buffaloes.

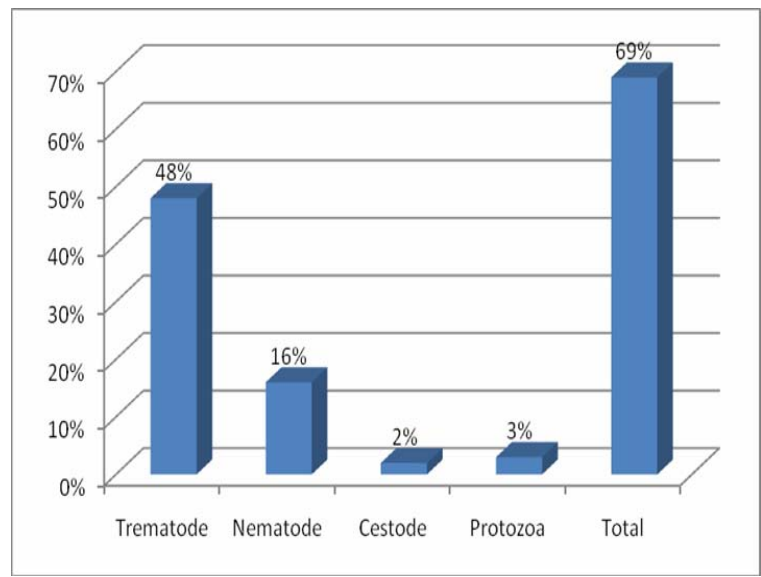

Fig.1 Overall incidences of parasites in buffaloes

Incidence of nematodes: Also the nematode infestation was statistically significant $(P<0.01)$. The highest infestation was of Oesophagostomum sp. (5\%) followed by Toxocara sp. (4\%), Bunostomum sp. (3\%), Cooperia sp. (2\%), Strongyloides sp (1\%) and Trichostronghylus sp. (1\%). Steel et al. (1997) reported that the gastrointestinal parasites were proportionately higher in buffalos. These results also support the earlier reports by Krishna et al. (1989) who found $2-6 \%$ incidence of Strongyloides sp. in water buffalos, and by Rahman et al. (2009) where prevalence of gastrointestinal parasites in buffaloes was higher.

Incidence of cestodes: Out of the 2 positive samples for cestodes, one genus (Monezia) was recorded with 2 species (2\%). Similar finding was reported by Wymann et al. (2006) who found 3\% cestode in buffaloes.

Incidence of protozoa: Several prorotozoan oocysts were found in buffalo faeces. Out of the 3 positive samples for protozoa, 2 genera (Balantidium and Coccidia) were observed with 2 species, constituting 3\% incidence. Similar results from faeces samples were reported earlier (Wymann et al., 2006; Akhter et al, 2001). 
Table 1 Incidence of gastrointestinal parasites in buffaloes.

\begin{tabular}{|c|c|c|c|c|}
\hline Classes & Parasitic species & $\begin{array}{l}\text { Positive samples (\%) } \\
\qquad N=100\end{array}$ & Mean \pm SD & P-values \\
\hline \multirow{4}{*}{ Trematoda } & Schistosoma sp & $23(23 \%)$ & \multirow{4}{*}{$16.00 \pm 6.56$} & \multirow{4}{*}{$.0001^{*}$} \\
\hline & Paramphistomum sp & $15(15 \%)$ & & \\
\hline & Fasciola sp & $10(10 \%)$ & & \\
\hline & Total & $48(48 \%)$ & & \\
\hline \multirow{7}{*}{ Nematoda } & Toxocara sp & $4(4 \%)$ & \multirow{7}{*}{$2.67 \pm 1.63$} & \multirow{7}{*}{$.0001^{*}$} \\
\hline & Oesophagostomum sp & $5(5 \%)$ & & \\
\hline & Cooperia sp & $2(2 \%)$ & & \\
\hline & Bunostomum sp & $3(3 \%)$ & & \\
\hline & Trichostrongylus sp & $1(1 \%)$ & & \\
\hline & Strongyloides Sp & $1(1 \%)$ & & \\
\hline & Total & $(16 \%)$ & & \\
\hline
\end{tabular}

${ }^{*} \mathrm{P}<0.01, \mathrm{SD}=$ standard deviation.

Mixed infections: Among 69 positive samples, 10 samples were found as mixed infections with 3-4 species in each sample. Among helminthes, the intensity of light infection was noted due to Schistosoma sp. with 5\%, Paramphistomum sp. $2 \%$ and other protozoan infection $3 \%$, indicating a very high incidence of worm infestations in buffaloes, though there was wide variation in worm infestations in buffaloes. It may be attributed to the fact that buffaloes are prone to internal parasites due to their habit of wallowing in marshy areas, from where they may have engulfed the eggs/larvae of different species of worms. The higher incidence of worm infestation may be attributed to the higher rainfall during the study period.

\section{References}

Akhter, N., Mohammad, I.R., Mirbahar, K.B., Memon, M.I. \& Soomro, S.A. 2001. Prevalence of helminthiasis in buffaloes in cattle colony Hyderabad. Online J. Biol. Sci. 1: 158-159.

Kabir, M.H.B., Eliyas, M., Hashem , M.A., Mohiuddin \& Miazi, O.F. 2010. Prevalence of zoonotic parasitic diseases of domestic animals in different abattoir of Comilla and Brahman Baria region in Bangladesh. Univ. j. zool. Rajshahi. Univ. 28: 21-25.

Krishna, L.K.P., Jithendran, P. \& Veid, J.1989. Incidence of common parasitic infestation among ruminants in Kangra Valley of Himachal Pardesh. Int. J. Anin. Sci. 2: 183-184.
Raza, A.M., Iqbal, Z., Jabbar, A. \& Yaseen, M. 2007. Point prevalence of gastrointestinal helminthiasis in ruminants in southern Punjab, Pakistan. J. Helminthol. 81: 323-328.

Rahman, M.A., Javed, K.K., Tunio, M.T. \& Kuthu, Z.H. 2009. Passive surveillance of gastrointestinal parasites in buffaloes of Bahauddin and Gujrat Districts of the Punjab. J. Animal. PInt. Sci. 19: 1719.

Shaikh, H.U., Haq. M.M., Karim, M.K. \& Khan, M.M.M. 1983. Parasites of zoonotic importance in domesticated ruminants. Pakistan Vet. J. 3:23-25.

Shan, H.L. \& Chaudhry, R.K. 1995. Parasitism in dairy animals in India: Present status and its impact. In: Proceedings of the workshop on control strategy against gastrointestinal parasites in dairy animals in India using medicated urea molasses blocks. (NDDB, An \&, India): 1-5.

Steel, R.G.D., Torrie, J.H. \& Dickey, D.A. 1997. Principles and Procedures of Statistics. A Biometrical Approach (3rd edn). McGraw Hill Book Co. New York, USA.

Tum, S., Puotinen, M.L., Skerratt, L.F., Chan, B. \& Sothoeun, S. 2007. Validation of a geographic information system model for mapping the risk of fasciolosis in cattle \& buffaloes. Cambodia Vet. Parasitol. 143: 364-367.

Wymann, M.N., Bonfoh, B., Traore, K., Tembely, S. \& Zinsstag, J. 2006. Species diversity and acquisition of gastrointestinal parasites in calves aged 0-13 months in peri urban livestock production in Mali. Vet. Parasitol. 143 (19): 67-73. 